\title{
Clinical significance of CDIO expression in cancer
}

\begin{abstract}
CD10 is a zinc-dependant metalloproteinase, the expression of which can be observed on numerous tissues such as epithelial cells of the lung, intestine, kidney, breast and placenta. Earlier research studies have shown, that CD10 expression is commonly observed in different types of carcinomas. Furthermore, there is an association between CD10 expression and tumor size and histological grade. Expression of CD10 might help with assessment of disease status, progression and prognosis.
\end{abstract}

Keywords: CD10, cancer, breast cancer, malignant melanoma, prostate cancer

\author{
Volume 5 Issue I - 2017
}

\author{
Joanna Włodek \\ Department of Clinical Immunology and Transplantology, \\ Jagiellonian University, Poland
}

Correspondence: Joanna Włodek, Department of Clinical Immunology and Transplantology Collegium Medicum Jagiellonian University, Poland,

Email joanna.wlodek9I@gmail.com

Received: July 28, 2017 | Published: August 18, 2017

\section{Introduction}

CD10 is common zinc-dependent metalloendo protease or membrane metalloendo peptidase located on the cell surface. Its main function is to inactivate a number of signaling peptides. ${ }^{1,2}$ Physiologically, CD10 is highly expressed on epithelial cells of lung, intestine, kidney, breast, placenta and many others. Initially, CD10 was identified as a tumor-specific antigen of leukemia cells, (common acute lymphoblastic leukemia antigen), ${ }^{3}$ following with detection of this determinant on early precursors of B-cell line in bone marrow. Since then, CD10 expression besides lymphoblastic leukemia and non-Hodgkin's lymphoma of B-cell line has also been observed in number of cancers as gastric, lung, breast prostate and colorectal. The observations showed correlation between level of CD10 expression and higher histological grade, larger tumor size, vascular invasion and overall survival rate patients with solid tumors. ${ }^{4}$

High CD10 expression has been noted in prostate, follicular papillary and papillary thyroid cancer, melanomas, and esophageal carcinoma. In contrast, cervical carcinoma and adenocarcinoma of the gastrointestinal tract have shown a decrease in the expression of CD10. The clinical significance of this expression is different, however, it seems important to considered CD10 expression as additional parameter for estimation of cancer therapy response and prognosis. ${ }^{5}$ Current methods of cancer diagnosis consist of numerous tests and procedures, which allow to assess the disease progression, qualification of patients to the appropriate risk group and implementation of the correct treatment regimen. Current research shows, that CD10 may become a significant and uninfluenced predictive factor for tumor aggressiveness, therapy response and overall survival. ${ }^{6}$

\section{Breast cancer}

Breast cancer is the most frequent cancer diagnosed in woman all over the world. This life-threatening cancer is the leading cause of death in woman, ${ }^{7}$ despite the progress in prevention, diagnosis and therapy of this malignancy. Standard prognostic factors consist of tumor grade and lymph nodes involvement and molecular markers (estrogen and progesterone receptors, HER-2/neu expression) associated with invasiveness, metastatic potency and therapy response. ${ }^{8,9}$ Introduction of a new independent marker could be helpful in management of patients including modification of therapy schedule. Recent studies have shown strong correlations between CD10 expression in stromal cells and stage of disease. CD10 expression is only physiologically present in the stromal myoepithelial cells of normal breast tissue. ${ }^{10-12}$ In case of invasive breast carcinoma, the CD10 expression in stromal cells surrounding the infiltrating tumor cells was observed 6 . When cancer cells invade and metastasize, the number of stromal cells increases. The CD10 positive stromal cells are present at the cancer cells invasive front suggests the interaction between breast cancer cells and CD10-positive stromal cells. Moreover, presence of CD10 on stromal cells positively correlates with nodal metastasis, tumor grade, tumor size, lymph node and worsening prognosis. ${ }^{13}$

\section{Malignant melanoma}

Malignant melanoma (MM) is an aggressive skin cancer characterized by dermal infiltration and destruction of skin appendages, vessels and stromal fibroblasts. The prognosis for majority of patients is poor. MM is a uncontrolled proliferation of pigment-producing cells (melanocytes) occurring mainly in the skin, but also in eyes, gastrointestinal tract or ears. ${ }^{14,15}$ Diagnosis based on clinical and pathological findings in skin biopsy have gradually improved in last years. However, despite advanced techniques such as dermatoscopy, immunochemistry (IHC) or molecular analyses of bioptic materials, the problems of precise differential diagnosis of skin abnormalities are still present. Moreover, the majority of MM cases are diagnosed without difficulty, some cases, like MMs with equivocal invasiveness (in situ lesions) or benign melanocytic nevi $(\mathrm{BMN})$, characterized by growth without damage of dermal texture still remained problematic. Current methods are very useful and standard, but diagnostic panels in the case of differentiating MM from BMN are still under discussion. ${ }^{16-19}$ Cases presenting wide dermal involvement in BMN are difficult to recognize. Research studies shown CD10 expression in MMs as lower in primary tumor $(21,4 \%)$ than in metastatic lesions in skin $(69,9 \%)$ or lymph nodes $(61,0 \%) .{ }^{20}$ In consequence, up-regulation of CD10 expression in association with tumor progression was observed in Hoshikawa MM at al. ${ }^{21}$ found CD10 expression in $94,4 \%$ of MM samples (in tumor and stromal cells) compared with $16 \%$ of stromal cells in BMN. These results suggest the possible role of CD10 as a useful marker of malignancy helping in difficult differential diagnosis. 


\section{Prostate cancer}

The cancer of prostate is most frequent cancer in men. Advanced diagnostic techniques and improvement of therapy schedule reduced mortality caused by this type of solid tumor. Common data underline the importance of prevention, early disease detection and improvement of diagnostic and therapy procedures. ${ }^{22,23}$ Assessment of Prostate Specific Antigen (PSA) is commonly used parameter in control of the effectiveness and results of treatment. ${ }^{24,25} \mathrm{CD} 10$ shows high expression in normal prostatic luminal epithelial cells, but this expression in cancer cases present different pattern. ${ }^{26}$ In many patients with primary tumors, the expression of CD10 was heterogeneous. Furthermore, tumors in early stages present decline of CD10 expression. ${ }^{27}$ The up-regulation of CD10 expression and an advanced Gleason score showed by Dall'Era et al. ${ }^{28,29}$ Is associated with worse prognosis and poor response to therapy. In patients demonstrating high expression of CD10 on the primary tumor cells, the lymph node metastasis and relapse (rise of PSA after radically prostatectomyRP) occur more often. However, in contrast to this result, Osman at al. ${ }^{30}$ showed association between decline of CD10 expression and worse treatment outcome. In this group of patients the loss of CD10 expression correlated with PSA rise after RP. These discrepancies in results may depend on other reference points and the different characteristics of studied groups of patients, so these two studies are difficult to compare.

\section{Conclusion}

Expression of CD10 on tumor or stromal cells may be used as prognostic marker in selected cancers. Additionally to describe examples of cancers, different CD10 expression in renal, urinary bladder and colorectal cancers is observed. ${ }^{31-33}$ Potentially, the inhibition of CD10 expression may be used as supplementary therapy in given solid tumors.

\section{Acknowledgements}

None.

\section{Conflict of interest}

The author declares no conflict of interest.

\section{References}

1. Roques BP, Noble F, Dauge V. Neutral endopeptidase 24.11: Structure, inhibition, and experimental and clinical pharmacology. Pharmacol Rev. 1993;45(1):87-146

2. Turner AJ, Tanzawa K. Mammalian membrane metallopeptidases: NEP, ECE, KELL, and Pex. FASEB J. 1997;11(5):355-364.

3. Maguer-Satta V. Concise review: neutral endopeptidase (CD10): a multifaceted environment actor in stem cells, physiological mechanisms, and cancer. Stem Cells. 2011;29(3):389-396.

4. Kim HS, Kim GY, Kim YW, et al. Stromal CD10 expression and relationship to the E-cadherin/beta-catenin complex in breast carcinoma. Histopathology. 2010;56(6):708-719.

5. Mishra D, Singh S, Narayan G. Role of B cell development marker CD10 in cancer progression and prognosis. Molecular Biology International. 2016;9:1-9.

6. Iwaya K, Ogawa H, Izumi M, et al. Stromal expression of CD10 in invasive breast carcinoma: a new predictor of clinical outcome. Virchows Arch. 2002;440(6):589-593.
7. Sharma G, Dave R, Sanadya J. Various types and management of breast cancer: an overview. Adv Pharm Technol Res. 2010;1(2):109-126.

8. Gonzalez-Angulo AM, Hortobágyi GN, Esteva FJ. Adjuvant therapy with trastuzumab for HER-2/neu-positive breast cancer. Oncologist. 2006;11(8):857-867.

9. Ménard S, Fortis S, Castiglioni F. HER2 as a prognostic factor in breast cancer. Oncology. 2001;61:67-72.

10. Kalof AN, Tam D, Beatty B. Immunostaining patterns of myoepithelial cells in breast lesions: acomparison of CD10 and smooth muscle myosin heavy chain. J Clin Pathol. 2004;57(6):625-629.

11. Moritani S, Kushima R, Sugihara H. Availability of CD10 immunohistochemistry as a marker of breast myoepithelial cells on paraffin sections. Mod Pathol. 2002;15(4):397-405.

12. Popnikolov NK, Ayala AG, Graves K. Benign myoepithelial tumors of the breast have immunophenotypic characteristics similar to metaplastic matrixproducing and spindle cell carcinomas. Am J Clin Pathol. 2003;120(2):161-167.

13. Anuradha Devi BV, Chandra Sekhar S, Saritha C. A study on stromal CD10 expression in invasive breast carcinoma. IAIM. 2016;3(6):142-147.

14. Cancer Research UK. Skin cancer incidence statistics. UK: Cancer Research; 2014.

15. Weinstein D, Leininger J, Hamby C. Diagnostic and prognostic biomarkers in melanoma. J Clin Aesthet Dermatol. 2014;7(6):13-24.

16. Ivan D, Prieto VG. Use of immunohistochemistry in the diagnosis of melanocytic lesions: applications and pitfalls. Future Oncol. 2010;6(7):1163-1175.

17. Ordonez NG. Value of melanocytic-associated immunohistochemical markers in the diagnosis of malignant melanoma: a review and update. Hum Pathol. 2014;45(2):191-205.

18. Chandler WM, Rowe LR, Florell SR. Differentiation of malignant melanoma from benign nevus using a novel genomic microarray with low specimen requirements. Arch Pathol Lab Med. 2012;136(8):947-955.

19. Ohsie SJ, Sarantopoulos GP, Cochran AJ. Immunohistochemical characteristics of melanoma. J Cutan Pathol. 2008;35(5):433-444.

20. Kanitakis J, Narvaez D, Claudy A. Differential expression of the CD10 antigen (neutral endopeptidase) in primary versus metastatic malignant melanomas of the skin. Melanoma Res. 2002;12(3):241-244.

21. Hoshikawa M, Koizumi H, Handa R. Immunohistochemical CD10 expression is useful for differentiating malignant melanoma from benign melanocytic nevus. J St Marianna Univ. 2015;6(2):111-118.

22. Mottet N, Schalken J, Heidenreich A. Highlights on prostate cancer from urological and oncological congresses in 2007. 2007;7(6):460-476.

23. Stangelberger A, Waldert M, Djavan B. Prostate cancer in elderly men. Rev Urol. 2008;10(2):111-119.

24. Loeb S, Roehl K, Catalona W. Prostate specific antigen velocity threshold for predicting prostate cancer in young men. J Urol. 2007;177(3):899902.

25. Gjertson CK, Albertsen PC. Use and assessment of PSA in prostate cancer. Med Clin North Am. 2011;95(1):191-200.

26. Goo YA, Goodlett DR, Pascal LE, et al. Stromal mesenchyme cell genes of the human prostate and bladder. BMC Urology. 2005;5:17.

27. Freedland SJ, Seligson DB, Liu AY, et al. Loss of CD10 (neutral endopeptidase) is a frequent and early event in human prostate cancer. Prostate. 2003;55(1):71-80. 
28. Dall'Era M, True L, Siegel A. Differential expression of CD10 in prostate cancer and its clinical implication. BMC Urology. 2007;7:3.

29. Chen N, Zhou Q. The evolving Gleason grading system. Chin J Cancer Res. 2016;28(1):58-64.

30. Osman I, Yee H, Taneja SS, et al. Neutral endopeptidase protein expression and prognosis in localized prostate cancer. Clin Cancer Res. 2004;10(12 Pt 1):4096-4100.

31. Wenjuan Y, Yuewei W. Distinct immunophenotypes and prognostic factors in renal cell carcinoma with sarcomatoid differentiation: a systematic study of 19 Immunohistochemical markers in 42cases. $B M C$ Cancer. 2017;17(1):293.
32. Mohammed A, Ali H. CD10 and CA19.9 immunohistochemical expression in transitional cell carcinoma of the urinary bladder. Urol Ann. 2013;5(2):81-85.

33. Levindo Alves de Oliveira, Ricardo Artigiani Neto. Tissue expression of CD10 protein in colorectal carcinoma: correlation with the anatomopathological features of the tumor and with lymph node and liver metastases. Journal of Coloproctology. 2012;32(1). 